\section{Conhecimento sobre atividade física e fatores associados em adolescentes estudantes do ensino médio da zona rural}

\author{
Knowledge about physical activity and \\ associated factors among secondary \\ adolescent students living in the rural area
}

Fábio da Conceição de Barros ${ }^{1}$

Marcelo Cozzensa da Silva ${ }^{1,2}$

\section{Resumo}

O presente estudo avaliou o conhecimento sobre atividade física (AF) e fatores associados de adolescentes do ensino médio de escolas da zona rural da região sul do Rio Grande do Sul. Foi realizado um estudo transversal de base escolar com 510 adolescentes que responderam a um questionário com questões fechadas sobre os benefícios da atividade física para a saúde, para a prevenção de doenças crônicas, finalidades da atividade física e efeitos da mesma no organismo humano. O desfecho foi analisado de forma contínua (0 a 58 pontos). A média de acertos foi de 35,8. Indivíduos do sexo feminino de cor de pele branca e ativos fisicamente, assim como adolescentes de ambos os sexos com maior nível de escolaridade apresentaram maiores médias de conhecimento sobre à pratica de atividade física e seus benefícios. Com base nos achados, programas de educação para a saúde devem ser promovidos aos jovens na tentativa de ajudar a minimizar o sedentarismo e suas morbidades associadas.

\section{Palavras-chave}

Conhecimento, Atividade motora, Adolescente, Educação, Comunidade Rural.

\begin{abstract}
This study assessed the knowledge about physical activity $(P A)$ and associated factors of adolescents from high schools in rural southern Rio Grande do Sul. It was conducted a cross-sectional school-based study with 510 adolescents who responded a questionnaire with closed questions about the benefits of physical activity for health, for the prevention of chronic diseases, physical activity goals and effects of the same in the human body. The outcome was analyzed continuously (0-58). The mean score was 35.8. Females of white skin color and physically active, as well as adolescents of both sexes with higher level of education had higher average knowledge about the practice of physical activity and its benefits. Based on the findings, programs of health education should be promoted for young people in an effort to help minimize physical inactivity and its associated morbidities.
\end{abstract}

\section{Keywords}

Knowledge, Motor Activity, Adolescent, Education, Rural Community.
Rev Bras Ativ Fis Saúde p. 594-603

DOI:

http://dx.doi.org/10.12820/rbafs.v.18n5p594

1 Escola Superior de Educação Física, Universidade Federal de Pelotas

2 Grupo de Estudos em Epidemiologia da Atividade Física (GEEAF) 


\section{INTRODUÇÃO}

A atividade física vem ganhando cada vez mais destaque na mídia como um dos principais fatores responsáveis pela melhoria da saúde e da qualidade de vida. Notadamente existe uma quantidade imensa de informação, tanto veiculadas pelos meios de comunicação quanto por artigos científicos, sobre os seus benefícios da mesma à saúde ${ }^{1,2}$. Pesquisas afirmam que a população, de forma geral, reconhece a importância da atividade física na prevenção e/ou tratamento de várias doenças $^{3,4}$, mas apesar de haver atualmente muitas campanhas e programas de televisão mostrando a importância de se adquirir hábitos saudáveis, o número de pessoas sedentárias na última década vem aumentando 5 .

Nahas ${ }^{6}$ descreve que a inatividade física representa uma causa importante de debilidade, de reduzida qualidade de vida e morte prematura nas sociedades contemporâneas, particularmente nos países industrializados. Nesse sentido, atividade física regular tem grande importância na saúde da população, tanto na prevenção de doenças crônicas, como na interação social, pois muitos vêm nos programas de atividade física, além de um meio de aprimorar a saúde, uma maneira de conhecer e interagir com outras pessoas ${ }^{7,3}$.

Lazzoli et $a 1 .{ }^{8}$, descrevem que a atividade física na adolescência contribui para melhoria no perfil lipídico e metabólico, e também na redução do percentual de gordura corporal. Tassitano et al. ${ }^{9}$, salientam que a atividade física apresenta grandes benefícios na saúde dos ossos nessa fase da vida. Guedes et al. ${ }^{10}$, afirmam que um jovem ativo fisicamente tem maiores chances de levar esses hábitos para vida adulta, momento da vida onde as doenças crônicas começam a aparecer.

Pesquisas em diversas áreas, principalmente os estudos sobre o comportamento humano, têm revelado que o conhecimento sobre uma determinada questão, como por exemplo, o hábito de fumar ou a prática de exercícios, está relacionado com a atitude que uma pessoa pode tomar diante desta questão ${ }^{6}$. De fato, observa-se que pessoas com um maior conhecimento sobre os benefícios da prática de exercícios físicos normalmente são mais ativas fisicamente ${ }^{6}$.

Partindo dessa premissa, o estudo buscou verificar o conhecimento sobre atividade física e fatores associados em estudantes do ensino médio da zona rural da região sul do Rio Grande do Sul.

\section{METODOLOGIA}

A presente pesquisa caracteriza-se como sendo observacional de corte transversal do tipo censo. Foram convidados a participar do estudo todos os estudantes do $1^{\circ}, 2^{\circ}$ e $3^{\circ}$ anos das escolas da zona rural de Ensino Médio, compreendidas pela $5^{\circ}$ Coordenadoria Regional de Educação, mas só foram incluídos na população aqueles com idade entre 13 e 19 anos de idade. Dez escolas se enquadraram nos requisitos, somando um total de 1502 alunos. Das escolas estudadas quatro estavam localizadas em Canguçu, duas em São Lourenço do Sul, duas em Pelotas, uma em Piratini e uma em Cerrito.

Foi solicitada, junto à 5a Coordenadoria Regional de Educação (CRE), uma autorização para a realização da pesquisa nas escolas. Houve um contato preliminar com cada escola para saber se havia interesse em participar do estudo e explicar os objetivos dos mesmos. Na primeira visita realizada foram explicitados a cada turma os objetivos da pesquisa e entregue a cada aluno o Termo de Consentimento Livre e Esclarecido. Na segunda visita foi aplicado o questionário e realizada a verificação do peso corporal e da estatura dos alunos. 
O instrumento utilizado para a coleta de dados foi um questionário contendo perguntas sobre características socioeconômicas, demográficas, comportamentais, nutricionais e de saúde, além de questões específicas de conhecimento sobre atividades físicas. $\mathrm{O}$ objetivo das questões sobre conhecimento foi verificar os benefícios da atividade física para a saúde, para a prevenção de doenças crônicas, finalidades da atividade física e seus efeitos no organismo humano. A mensuração do peso e estatura foi realizada por medida direta, respectivamente, por meio de uma balança da marca Slim Control, com capacidade $150 \mathrm{~kg}$ e com precisão de $100 \mathrm{~g}$, e fita métrica metálica inextensível, tamanho $150 \mathrm{~cm}$ e com precisão de $1,0 \mathrm{~mm}$.

As perguntas sobre conhecimento foram baseadas nos questionários de Silveira e Silva ${ }^{11}$, Borges et al. ${ }^{12}$ e Domingues et al. ${ }^{13}$, os quais vem sendo utilizados pela comunidade científica, bem como questões formuladas pelos próprios pesquisadores. A definição de certo ou errado para cada resposta baseou-se em diversos artigos sobre associação entre atividade física e saúde e do efeito da atividade física no organismo humano ${ }^{14,15,16}$.

Com base nos dados do questionário foi possível gerar dois conjuntos de informação: um escore de conhecimento e uma descrição dos itens pesquisados. $\mathrm{O}$ escore foi obtido por meio das respostas e a pontuação total poderia variar entre 0 (zero) e 58 (cinquenta e oito). Respostas erradas não diminuíam a pontuação, apenas não acrescentavam pontos ao escore. Cada uma das questões que compunham o instrumento para a avaliação do conhecimento apresentava o mesmo peso no escore de conhecimento.

Os dados foram duplamente digitados utilizando o programa Epi Info 6.0, com checagem automática de consistência, para correção de possíveis erros. A análise dos dados foi realizada no programa estatístico Stata 12.0.

A análise descritiva caracterizou a amostra total e estratificada por sexo, conforme variáveis socioeconômicas, comportamentais, de saúde e de conhecimento. $\mathrm{Na}$ análise bruta, a associação entre as variáveis independentes e o desfecho (contínuo) foi realizada por meio do teste de tendência linear para as variáveis com mais de duas categorias ordenadas, e para as de duas categorias o teste de heterogeneidade de médias.

O projeto foi submetido e aprovado pelo Comitê de Ética em Pesquisa da Faculdade de Educação Física da Universidade Federal de Pelotas (Protocolo número 038/2011). A coleta de dados foi efetuada após esclarecimento sobre o propósito da pesquisa e o termo de consentimento assinado pelos responsáveis dos alunos. O sigilo das informações e o direito de recusa foram garantidos aos estudantes.

\section{RESULTADOS}

Foram estudados 510 alunos de 13 a 19 anos matriculados no Ensino Médio, na zona rural, das escolas da 5 a $\mathrm{CRE}$. Cinquenta e nove por cento dos alunos eram do sexo feminino, com idade média de 16,3 anos $(\mathrm{DP}=1,2)$, na grande maioria de cor da pele branca (92,3\%). Em relação a escolaridade dos alunos, 37,7\% estavam no primeiro ano do ensino médio, $30,1 \%$ no segundo ano e $32,2 \%$ alunos no terceiro ano. Quanto ao nível de atividade física no lazer, 53,3\% foram considerados fisicamente ativos e, em relação ao IMC, menos de $17 \%$ encontravam-se nas categorias referentes à sobrepeso/obesidade (Tabela 1). A média do escore de conhecimento dos adolescentes foi de 35,8 pontos, atingida por $62 \%$ do total dos indivíduos.

$\mathrm{Na}$ tabela 2 podem ser encontradas questões fechadas de múltipla escolha, estratificadas por sexo e para toda população mostrando a frequência de respostas obtidas para algumas das variáveis avaliadas. Verificou-se que aproximadamente de $60 \%$ dos adolescentes associaram a atividade física com a prevenção de doenças 
Tabela 1 - Descrição das variáveis demográficas, antropométricas e comportamentais de escolares pertencentes a $5^{\text {a }}$ CRE-RS do ensino médio da zona rural, 2012.

\begin{tabular}{|c|c|c|}
\hline Variáveis & $\mathrm{N}$ & $\%$ \\
\hline \multicolumn{3}{|l|}{$\operatorname{Sexo}(N=510)$} \\
\hline Masculino & 209 & 41,0 \\
\hline Feminino & 301 & 59,0 \\
\hline \multicolumn{3}{|l|}{ Idade $(\mathrm{N}=510)$} \\
\hline $13 / 14$ anos & 25 & 4,9 \\
\hline 15 anos & 131 & 25,7 \\
\hline 16 anos & 141 & 27,7 \\
\hline 17 anos & 125 & 24,5 \\
\hline $18 / 19$ anos & 88 & 17,2 \\
\hline \multicolumn{3}{|l|}{ Cor da pele $(\mathrm{N}=510)$} \\
\hline Branca & 454 & 92,3 \\
\hline Não branca & 38 & 7,7 \\
\hline \multicolumn{3}{|l|}{ Escolaridade $(\mathrm{N}=510)$} \\
\hline $1^{\circ}$ ano & 192 & 37,7 \\
\hline $2^{\circ}$ ano & 154 & 30,1 \\
\hline $3^{\circ}$ ano & 164 & 32,2 \\
\hline \multicolumn{3}{|c|}{ Tabagismo nos últimos 30 dias $(\mathrm{N}=510)$} \\
\hline Não & 465 & 92,6 \\
\hline Sim & 37 & 7,4 \\
\hline \multicolumn{3}{|c|}{ Consumo de álcool nos últimos 30 dias $(\mathrm{N}=510)$} \\
\hline Não & 167 & 33,1 \\
\hline Sim & 338 & 69,9 \\
\hline \multicolumn{3}{|c|}{ Nível de atividade física $(\mathrm{N}=510)$} \\
\hline Insuficientemente ativo & 238 & 46,7 \\
\hline Ativo & 272 & 53,3 \\
\hline \multicolumn{3}{|l|}{ IMC $(N=510)$} \\
\hline Normal & 422 & 82,9 \\
\hline Sobrepeso & 60 & 11,8 \\
\hline Obesidade & 27 & 5,3 \\
\hline
\end{tabular}

crônicas como pressão alta, colesterol elevado e depressão, mas foi baixo o número daqueles que associaram a atividade física na prevenção de osteoporose $(38,7 \%)$. Ainda percebeu-se que cerca de $90 \%$ dos alunos souberam que atividade física não apresenta relação alguma na prevenção de doenças como câncer de pele, AIDS e dor de estômago.

A maioria dos entrevistados afirmou corretamente que a caminhada contínua pode trazer benefícios como aumento do gasto de gordura corporal e redução do risco de infarto no miocárdio. Acima de $40 \%$ dos entrevistados afirmou que a caminhada também influencia na redução da pressão arterial de repouso e que esse tipo de atividade é o melhor exercício para perder gordura durante a prática. Menos de 6\% dos estudados respondeu incorretamente que a caminhada pode trazer benefícios como diminuição do risco de contrair HIV.

Apenas $15 \%$ souberam responder adequadamente qual a recomendação indicada de atividade física semanal para adolescentes para a obtenção de benefícios à saúde pela OMS. Um percentual de 28,1\% afirmou que atividades aeróbicas, de força muscular e flexibilidade, são as atividades mais indicadas para se buscar saúde e qualidade 
de vida e aproximadamente $40 \%$ dos alunos responderam de forma correta que, para melhorar a forma física através da corrida, deve-se, com o tempo, aumentar a sua duração e intensidade e que a frequência cardíaca diminui com a prática regular.

Tabela 2 - Percentual de respostas às questões relativas ao conhecimento sobre atividades físicas e fatores associados, para toda a população e estratificado por sexo, de escolares do ensino médio da zona rural pertencentes a 5a CRE-RS, 2012.

\begin{tabular}{|c|c|c|c|}
\hline \multirow[t]{2}{*}{ Variável } & \multicolumn{3}{|c|}{ Percentual de respostas referente às variáveis } \\
\hline & Todos & Homens & Mulheres \\
\hline \multicolumn{4}{|c|}{$\begin{array}{l}1 \text { - Quais doenças você considera que pode }(\mathrm{m}) \text { ser prevenidas com o hábito de fazer } \\
\text { atividade física?* }\end{array}$} \\
\hline Pressão Alta & 58,0 & 55,8 & 59,5 \\
\hline Câncer de pele & 4,7 & 4,8 & 4,6 \\
\hline Colesterol alto & 61,1 & 65,5 & 58,1 \\
\hline AIDS & 3,0 & 3,4 & 2,7 \\
\hline Osteoporose & 38,7 & 35,1 & 41,2 \\
\hline Dor de estomago & 10,8 & 8,6 & 12,3 \\
\hline Depressão & 67,4 & 68,3 & 66,8 \\
\hline \multicolumn{4}{|l|}{2 - Atividade física como caminhar, pode trazer quais benefícios? } \\
\hline Redução da pressão arterial de repouso & 41,3 & 42,3 & 40,5 \\
\hline Aumento do gasto de gordura corporal & 80,0 & 80,3 & 79,7 \\
\hline $\begin{array}{l}\text { Redução do risco de infarto do miocárdio } \\
\text { (doença do coração) }\end{array}$ & 74,5 & 72,6 & 75,7 \\
\hline Diminuição do risco de contrair HIV (AIDS) & 5,9 & 8,2 & 4,3 \\
\hline Todas as anteriores estão erradas & 9,4 & 8,2 & 10,3 \\
\hline \multicolumn{4}{|c|}{$\begin{array}{l}3 \text { - Atualmente, qual a recomendação de atividade física semanal orientada aos } \\
\text { adolescentes? }\end{array}$} \\
\hline Outras opções & 85,1 & 79,3 & 89,0 \\
\hline 300 minutos divididos na semana & 14,9 & 20,7 & 11,0 \\
\hline \multicolumn{4}{|c|}{$\begin{array}{l}\text { 4- Das seguintes atividades físicas abaixo, qual seria a melhor para uma pessoa } \\
\text { perder gordura durante a prática? }\end{array}$} \\
\hline Outras opções & 58,4 & 69,2 & 50,8 \\
\hline Caminhada continua & 41,6 & 30,8 & 49,2 \\
\hline \multicolumn{4}{|c|}{$\begin{array}{l}5 \text { - Para melhorar a forma física através da corrida, é necessário com o passar } \\
\text { do tempo: }\end{array}$} \\
\hline Outras Opções & 51,3 & 53,9 & 49,5 \\
\hline Aumentar a velocidade e duração da corrida & 48,7 & 46,1 & 50,5 \\
\hline \multicolumn{4}{|c|}{$\begin{array}{l}\text { 6- Quando praticamos atividade física e melhoramos nossa forma física, a } \\
\text { frequência cardíaca (batimentos do coração) quando estamos descansados: }\end{array}$} \\
\hline Outras opções & 62,7 & 59,1 & 65,1 \\
\hline Diminui em relação ao início do programa do exercício físico & 37,3 & 40,9 & 34,9 \\
\hline \multicolumn{4}{|c|}{$\begin{array}{l}7 \text { - Quais são as atividades mais indicadas para indivíduos que começam a se } \\
\text { exercitar buscando saúde e qualidade de vida? }\end{array}$} \\
\hline Outras opções & 71,9 & 68,8 & 74,1 \\
\hline Atividades aeróbicas, de força muscular e flexibilidade & 28,1 & 31,2 & 25,9 \\
\hline
\end{tabular}

* Percentual de adolescentes que respondeu positivamente a cada um dos ítens que compreendem a questão 1.

Na tabela 3 pode-se observar a média e o desvio padrão do escore de conhecimento sobre atividade física segundo as variáveis independentes incluídas no estudo. Além disso, são apresentadas as frequências dos escores divididas em quartis, segundo variáveis independentes. A única associação observada foi entre a variável escolaridade e os escores do desfecho (Tabela 3). 
Tabela 3 - Distribuição das variáveis sociodemográficas, comportamental e nutricional, segundo as categorias quartis de escores de conhecimento sobre atividade física e fatores associados, escolares pertencentes a $5^{\text {a }}$ CRE-RS do ensino médio da zona rural, 2012.

\begin{tabular}{|c|c|c|c|c|c|c|}
\hline Variável & $X( \pm D P)$ & $\begin{array}{l}0-33 \\
(\%)\end{array}$ & $\begin{array}{c}34-38 \\
(\%)\end{array}$ & $\begin{array}{c}39-41 \\
(\%)\end{array}$ & $\begin{array}{c}42-58 \\
(\%)\end{array}$ & Valor $\mathrm{p}$ \\
\hline \multicolumn{7}{|l|}{ Sexo } \\
\hline Masculino & $35,8(6,4)$ & 26,4 & 26,9 & 24,0 & 22,6 & \multirow[t]{2}{*}{0,8} \\
\hline Feminino & $35,8(6,5)$ & 28,2 & 27,6 & 20,6 & 23,6 & \\
\hline \multicolumn{7}{|l|}{ Cor da pele } \\
\hline Branco & $36,1(6,1)$ & 26,3 & 27,4 & 22,3 & 24,1 & \multirow[t]{2}{*}{0,4} \\
\hline N/Branco & $33,8(7,5)$ & 39,5 & 23,7 & 18,4 & 18,4 & \\
\hline \multicolumn{7}{|l|}{ Idade (anos completos) } \\
\hline $13-14$ & $35,5(5,0)$ & 16,7 & 45,8 & 20,8 & 16,7 & \multirow{5}{*}{0,5} \\
\hline 15 & $35,0(7,0)$ & 29,0 & 25,9 & 25,9 & 19,1 & \\
\hline 16 & $36,1(6,6)$ & 29,8 & 23,4 & 19,9 & 26,9 & \\
\hline 17 & $36,6(5,8)$ & 23,2 & 30,4 & 22,4 & 24,0 & \\
\hline $18-19$ & $35,5(6,8)$ & 30,7 & 26,1 & 19,3 & 23,9 & \\
\hline \multicolumn{7}{|l|}{ Escolaridade } \\
\hline $1^{\circ}$ ano ensino médio & $34,3(6,3)$ & 37,2 & 28,3 & 19,4 & 15,2 & \multirow{3}{*}{0,001} \\
\hline $2^{\circ}$ ano ensino médio & $36,2(7,0)$ & 23,5 & 27,5 & 24,8 & 24,2 & \\
\hline $3^{\circ}$ ano ensino médio & $37,2(5,9)$ & 20,1 & 26,2 & 22,6 & 31,1 & \\
\hline \multicolumn{7}{|l|}{ Atividade física } \\
\hline Insuficientemente ativos & $35,0(6,6)$ & 31,1 & 28,2 & 21,0 & 19,8 & \multirow[t]{2}{*}{0,2} \\
\hline Suficientemente ativos & $36,5(6,3)$ & 24,4 & 26,6 & 22,9 & 26,2 & \\
\hline \multicolumn{7}{|l|}{ IMC } \\
\hline Normal & $35,7(6,4)$ & 26,6 & 29,2 & 22,6 & 21,6 & \multirow{3}{*}{0,2} \\
\hline Sobrepeso & $36,5(6,8)$ & 33,3 & 18,3 & 15,0 & 33,3 & \\
\hline Obesidade & $35,9(6,0)$ & 29,6 & 18,5 & 25,9 & 25,8 & \\
\hline
\end{tabular}

Tabela 4 -Distribuição das variáveis sociodemográficas, comportamental e nutricional estratificadas por sexo, segundo os valores médios de conhecimento sobre atividade física e fatores associados, escolares pertencentes a $5^{\mathrm{a}}$ CRE-RS do ensino médio da zona rural, 2012.

\begin{tabular}{|c|c|c|c|c|}
\hline & \multicolumn{2}{|c|}{ Homens } & \multirow{2}{*}{$\frac{\text { Mulheres }}{x( \pm D P)}$} & \multirow{2}{*}{$\begin{array}{c}\text { Mulheres } \\
\mathrm{P}\end{array}$} \\
\hline Variável & $X( \pm D P)$ & $P$ & & \\
\hline \multicolumn{5}{|l|}{ Escolaridade } \\
\hline $1^{\circ}$ ano ensino médio & $34,4(6,2)$ & \multirow{3}{*}{$0,01 * *$} & $34,2(6,4)$ & \multirow{3}{*}{$0,003 * *$} \\
\hline $2^{\circ}$ ano ensino médio & $36,3(6,2)$ & & $36,1(7,4)$ & \\
\hline $3^{\circ}$ ano ensino médio & $37,4(6,5)$ & & $37,1(5,5)$ & \\
\hline \multicolumn{5}{|l|}{ Idade (anos completos) } \\
\hline $13-14$ & $37,7(2,8)$ & \multirow{5}{*}{0,5} & $33,9(5,6)$ & \multirow{5}{*}{0,05} \\
\hline 15 & $36,2(6,0)$ & & $34,1(7,6)$ & \\
\hline 16 & $34,7(6,3)$ & & $36,8(6,7)$ & \\
\hline 17 & $36,2(6,0)$ & & $36,5(5,7)$ & \\
\hline $18-19$ & $35,4(7,7)$ & & $35,8(5,4)$ & \\
\hline \multicolumn{5}{|l|}{ Cor da pele } \\
\hline Branco & $36,2(6,0)$ & \multirow[t]{2}{*}{0,3} & $36,1(6,2)$ & \multirow[t]{2}{*}{$0,04^{*}$} \\
\hline Não branco & $34,7(7,8)$ & & $33,1(7,4)$ & \\
\hline \multicolumn{5}{|l|}{ Nível de atividade física } \\
\hline Insuficientemente ativo & $35,0(5,9)$ & \multirow[t]{2}{*}{0,2} & $35,1(6,9)$ & \multirow[t]{2}{*}{$0,02 *$} \\
\hline Ativo & $36,2(6,6)$ & & $36,9(5,9)$ & \\
\hline \multicolumn{5}{|l|}{ IMC } \\
\hline Normal & $35,8(6,5)$ & \multirow{3}{*}{0,8} & $35,6(6,5)$ & \multirow{3}{*}{0,6} \\
\hline Sobrepeso & $36,3(6,8)$ & & $36,7(6,9)$ & \\
\hline Obesidade & $34,9(5,1)$ & & $36,9(6,9)$ & \\
\hline
\end{tabular}

* teste T para heterogeneidade de médias ** Teste de tendência linear. 
$\mathrm{Na}$ tabela 4 encontram-se os resultados médios do escore de conhecimento por categoria das variáveis independentes, bem como as associações entre o escore e as variáveis em estudo, separadamente para meninos e meninas. Algumas associações encontradas foram diferentes para homens e mulheres. A medida que aumentou o nível de escolaridade, tanto em meninos como em meninas, aumentou a média de conhecimento sobre atividade física e fatores associados. Meninas de cor da pele branca e consideradas ativas apresentaram médias superiores de conhecimento quando comparadas a seus pares.

\section{DISCUSSÃO}

O presente estudo demonstrou que mais de $50 \%$ dos adolescentes entrevistados foram considerados ativos fisicamente, valor superior ao encontrado por diversos estudos com adolescentes no Brasil ${ }^{17}$ e, especialmente, na cidade de Pelotas ${ }^{11,18,19}$. Uma hipótese para tal diferença talvez tenha sido a região em que os alunos moravam, tendo em vista que o presente estudo foi realizado apenas com adolescentes da zona rural. Muitos desses adolescentes relataram trabalhar na lavoura e percorrer grandes distâncias caminhando ou de bicicleta, o que pode ajudar a explicar a diferença encontrada nos estudos de comparação realizados com adolescentes da zona urbana.

Em relação ao IMC, a prevalência de sobrepeso/obesidade encontrada é inferior a descrita nos estudos de Silveira e Silva ${ }^{11}$ e Copetti et al. ${ }^{18}$ com adolescentes residentes da zona urbana. Tal diferença pode estar relacionada ao maior número de indivíduos que atingiram as recomendações de atividade física suficiente na zona rural e de uma provável alimentação mais adequada por eles consumida ${ }^{20}$.

A média do escore de conhecimento dos adolescentes ( 35,8 pontos) foi atingida por $62 \%$ do total dos entrevistados e é semelhante a encontrada por Silveira e Silva ${ }^{11}$ em estudo com escolares adolescentes da zona urbana da cidade de Pelotas, e de Domingues et al. ${ }^{13}$, na população adulta da mesma cidade. Sugerindo como ponto de corte pelo menos $70 \%$ de acertos para considerar-se um bom conhecimento (ponto utilizado por muitas escolas para aprovação imediata nas disciplinas curriculares), 76,8\% dos estudantes não alcançariam tal meta. Assuntos relacionados aos benefícios da atividade física para saúde parecem, ainda, estar sendo pouco discutidos no ambiente escolar.

As questões sobre relação entre atividade física e prevenção de doenças crônicas como pressão alta, colesterol elevado e depressão apresentaram resultados semelhantes aos encontrados por Silveira e Silva ${ }^{11}$, com exceção da relação entre atividade física e osteoporose, onde o percentual de acerto $(38,7 \%)$ foi inferior ao do outro estudo (66,42\%). No estudo de Borges et al. ${ }^{12}$, mais de $80 \%$ dos entrevistados mostraram-se cientes das associações entre sedentarismo e infarto agudo do miocárdio; tabagismo e câncer de pulmão; consumo abusivo de álcool e cirrose; alimentação inadequada e diabetes. Associações inexistentes também foram corretamente identificadas por uma grande parcela dos respondentes, como, por exemplo, a ausência de associação dos fatores sedentarismo, tabagismo, alimentação inadequada e AIDS. Segundo o mesmo autor, apesar da relação entre atividade física e osteoporose estar bem consistente na literatura ${ }^{21,22}$, esse conhecimento não foi incorporado pela população em geral ${ }^{12}$.

Cerca de $80 \%$ dos estudantes respondeu corretamente que a caminhada pode trazer benefícios como aumento do gasto de gordura corporal e redução do risco de infarto no miocárdio, em contrapartida, apenas $40 \%$ afirmou que a caminhada, 
além de ajudar no gasto de gordura corporal, é o melhor exercício para se perder gordura durante a prática, e que a caminhada ajuda na redução da pressão arterial de repouso. Estudos tem demonstrado que a importância da caminhada, enquanto umas das atividades físicas mais praticadas pela população adulta, já é conhecido há alguns anos ${ }^{23,24,25}$. Entretanto, apesar da grande e reconhecida importância de seus efeitos benéficos à saúde ${ }^{26,21,27}$, ainda são poucos os estudos sobre a mesma, especialmente entre a população adolescente.

A recomendação voltada à prática suficiente de atividade física semanal para benefícios a saúde para os adolescentes $(300 \mathrm{~min} / \mathrm{sem}$ ) difere da predita para os adultos (150 $\mathrm{min} / \mathrm{sem})$. Essa informação não parece estar devidamente difundida nesses indivíduos, visto que apenas $14,9 \%$ dos estudantes elegeu a resposta correta. Parece que a recomendação de atividades aeróbicas para adultos está mais arraigada ao conhecimento desses adolescentes ${ }^{11}$.

Chama a atenção a desinformação sobre os processos orgânicos desencadeados pela atividade física. Nessa população, mais de $60 \%$ dos adolescentes desconhecem que a frequência cardíaca diminui em relação ao início do programa do exercício físico, trazendo em benefício do praticante um menor esforço cardíaco para a uma mesma intensidade de exercício.

Indivíduos com maior nível de escolaridade apresentaram maior conhecimento sobre o assunto, resultado esse, corroborado pelos estudos Silveira e Silva ${ }^{11}$, Borges et al. ${ }^{12} \mathrm{e}$ Domingues et al. ${ }^{12}$ Estudos epidemiológicos mostram que, quanto maior a escolaridade, mais facilmente os indivíduos modificam seus comportamentos ${ }^{28,29}$.

A cor de pele branca e a prática de atividade física estiveram associados a maiores médias de conhecimento para as adolescentes do sexo feminino. A associação com a cor branca é a mesma encontrada por Silveira e Silva ${ }^{11}$ e Borges et al. ${ }^{12}$.Já a associação entre prática e conhecimento vai de encontro aos achados de Silveira e Silva ${ }^{11}$ e Domingues et al. ${ }^{13}$, e pode ter sido afetada pelo viés de causalidade reversa, isto é, os indivíduos são ativos porque conhecem mais ou conhecem porque praticam.

Alguns pontos devem ser descritos sobre o estudo. $\mathrm{O}$ mesmo utilizou um delineamento do tipo transversal, o qual é passível de acontecimento de viés de causalidade reversa. Isso pode ter acontecido na relação entre conhecimento e prática, no qual não se teve capacidade de determinar qual deles precede o outro. Outra limitação do estudo é o fato do instrumento não ter sido validado anteriormente. Apesar disso, o referido instrumento foi constituído por uma série de perguntas existentes em outros questionários sobre conhecimento amplamente utilizados em estudos epidemiológicos. As perguntas desenvolvidas pelos pesquisadores passaram por estudo piloto com o intuito de verificar a qualidade de entendimento em uma amostra semelhante a estudada.

\section{CONCLUSÕES}

O presente estudo identificou uma média de conhecimento de 35,8 acertos, o que corresponde acima de $60 \%$ do total de acertos. Em torno de $50 \%$ dos estudados atingiu valores acima do escore médio. Indivíduos do sexo feminino de cor de pele branca e ativos fisicamente, assim como adolescentes de ambos os sexos com maior nível de escolaridade apresentaram maiores médias de conhecimento sobre à pratica de atividade física e seus benefícios.

A participação do profissional da área da saúde, em especial dos professores de educação física, na difusão da importância da atividade física, bem como no planeja- 
mento é execução de aulas práticas que aumentem o conhecimento e prática de seus alunos é de fundamental importância na ajuda à prevenção de doenças crônicas.

\section{Contribuição dos autores}

Fábio da Conceição de Barros coordenou o trabalho de campo, conduziu a revisão de literatura e realizou a escrita do artigo. Marcelo Cozzensa da Silva idealizou o estudo, conduziu a análise de dados e revisão crítica da escrita do artigo.

\section{Agradecimentos}

À 5a Coordenadoria Regional de Educação - Pelotas, Rio Grande do Sul, aos diretores, professores e alunos participantes.

\section{REFERÊNCIAS}

1. Dunton GF, Schneider M. Perceived barriers to walking for physical activity. Prev Chronic Dis. 2006; 3:A116.

2. Coll C, Amorim TEC, Hallal PC. Percepção de adolescentes e adultos referente à influência da mídia sobre o estilo de vida. Rev Bras Ativ Fis e Saude. 2010; 15:105-10.

3. Domingues MR. Conhecimento populacional sobre atividade física. In: Florindo AA, Hallal PC (eds). Epidemiologia da Atividade Física. São Paulo: Editora Atheneu; 2011. p.139-150.

4. Knuth GA, Bielemann MR, Silva GS, Borges TT, Del Duca GF, Kremer MM, et al. Conhecimento de adultos sobre o papel da atividade física na prevenção e tratamento do diabetes e hipertensão: estudo de base populacional no sul do Brasil. Cad Saúde Pública. 2009; 25: 513-20.

5. Barreto MS, Pinheiro ARO, Sichieri R, Monteiro CA, Batista Filho M, Schimidt MI, et al. Análise da Estratégia Global para Alimentação, Atividade física e Saúde, da Organização Mundial da Saúde. Epidemiol Serv Saúde. 2005; 14: 41- 68

6. Nahas M. Atividade Física, Saúde e qualidade de vida: Conceitos e sugestões para um estilo de vida ativo. Londrina: Midiograf, 2010.

7. Franchi KMB, Montenegro RM. Atividade Física: Uma necessidade para a boa saúde na terceira idade. Rev Bras Promoção Saude. 2005; 18: 152-56.

8. Lazzoli JK, Nóbrega ACL, Carvalho T, Oliveira MAB, Teixeira JAC, Leitão MB, et al. Atividade física e saúde na infância e adolescência. Rev Bras Med Esporte. 1998; 4: 107-09.

9. Tassitano RM, Bezerra J, Tenório MCM, Colares V, Barros MVG, Hallal PC. Atividade física em adolescentes Brasileiros: Uma revisão sistemática. Rev Bras Cineantropom Desempenho Hum. 2007; 9: 55-60.

10. Guedes DP, Guedes ERP, Barbosa DS, Oliveira JA. Níveis de prática de atividade física habitual em adolescentes. Rev Bras Med Esporte. 2001; 7:187-99.

11. Silveira EF, Silva MC. Conhecimentos sobre atividade física em estudantes de uma cidade do sul do Brasil. Motriz. 2011; 17:456-67.

12. Borges TT, Rombaldi AJ, Knuth AG, Hallal PC. Conhecimento sobre fatores de risco para doenças crônicas: estudo de base populacional. Cad Saúde Pública. 2009; 25: 1511-20.

13. Domingues MR, Araújo CLP, Gigante DP. Conhecimento e percepção sobre exercício físico em uma população adulta urbana do sul do Brasil. Cad Saúde Pública. 2004; 20(1): 204-215.

14. Fagherazzi S, Dias RL, Bortolon F. Impacto do exercício físico isolado e combinado com dieta sobre os níveis séricos de HDL, LDL, colesterol total e triglicerídeos. Rev Bras Med. Esporte 2008; 14: 381-86.

15. Siqueira FV, Facchini LA, Azevado MR, Reichert FF, Bastos JP, Silva MC, et al. Prática de atividade física na adolescência e prevalência de osteoporose na idade adulta. Rev Bras Med. Esporte 2009; 15: 27-30.

16. Moraes H, Deslandes A, Ferreira C, Pompeu FAMS, Ribeiro P, Laks J. O exercício físico no tratamento da depressão em idosos: revisão sistemática. Rev Psiquiatr. RS 2007; 29: 70-79.

17. Farias Jr. JC. Associação entre prevalência de inatividade física e indicadores de condição socioeconômica em adolescentes. Rev Bras Med. Esporte 2008; 14:109-14.

18. Copetti J, Neutzling MB, Silva MC. Barreiras à prática de atividades físicas em adolescentes de uma cidade do sul do Brasil. Rev Bras Ativ Fis e Saude. 2010; 15: 88-94. 
19. Bastos JP, Araújo CLP, Hallal PC. Prevalence of insufficient physical activity and associated factors in Brazilian adolescents. J Phys Act Health. 2008; 5:777-94.

20. Ministério da Saúde. Secretaria de Atenção à Saúde. Coordenação-Geral da Política de Alimentação e Nutrição. Guia alimentar para a população brasileira: promovendo a alimentação saudável. Brasília: Ministério da Saúde, 2005.

21. Bauman AE. Updating the evidence that physical activity is good for health: an epidemiological review 2000-2003. J Sci Med Sport. 2004; 7: 6-19.

22. Pitanga FJ. Epidemiologia, atividade física e saúde. Rev Bras Ciênc Mov. 2002; 10:49-54.

23. Eyler AA, Brownson RC, Bacak SJ, Housemann RA. The epidemiology of walking for physical activity in the United States. Med Sci Sports Exerc. 2003; 35: 1529-36.

24. Bates JH, Janet HB, Serdula MK, Jones DA, Gillespie C, Ainsworth BE. Total and leisuretime walking among U.S. adults should every step count? Am J Prev Med. 2005; 29: 46-50.

25. Tudor - Locke C, van der Ploeg HP, Bowles HR, Bittman M, Fisher K, Merom D, et al. Walking behaviours from the 1965-2003 American Heritage Time Use Study (AHTUS). Int J Behav Nutr Phys Act. 2007; 27:1-9

26. Haskell WL, Lee IM, Pate RR, Powell KE, Blair SN, Franklin BA, et al. Physical activity and public health: updated recommendation for adults from the American College of Sports Medicine and the American Heart Association. Circulation. 2007; 116:1081-93.

27. Lee IM, Buchner DM. The importance of walking to public health. Med Sci Sports Exerc. 2008; 40: 512-8.

28. Camões M, Lopes C. Fatores associados à atividade física na população portuguesa. Rev Saúde Pública. 2008; 42: 208-16.

29. Pitanga FJG, Lessa I. Prevalência e fatores associados ao sedentarismo no lazer em adultos. Cad Saúde Pública. 2005; 21: 870-77.

$$
\begin{array}{r}
\text { Endereço para Correspondência } \\
\text { Marcelo Cozzensa da Silva } \\
\text { Rua Luís de Camões, } 625 \text { - Três Vendas; } \\
\text { Pelotas - RS, } 96055-630 \\
\text { (53) } 3273-2752 \\
\text { e-mail: cozzensa@terra.com.br }
\end{array}
$$

Recebido 29/10/2013

Revisado 21/11/2013

Aprovado 22/11/2013 